\title{
Biphasic effect of Max on Myc cotransformation activity and dependence on amino- and carboxy-terminal Max functions
}

\author{
George C. Prendergast, ${ }^{1,2}$ Robert Hopewell, ${ }^{1}$ Beverly J. Gorham, ${ }^{1}$ and Edward B. Ziff ${ }^{1,3}$ \\ ${ }^{1}$ Howard Hughes Medical Institute, Kaplan Cancer Center, and Department of Biochemistry, New York University \\ Medical Center, New York, New York 10016 USA; ${ }^{2}$ Department of Cancer Research, Merck Research Laboratories, Merck \\ and Co., West Point, Pennsylvania 19486 USA
}

In Ras cotransformation assays, Max exhibited a biphasic effect on Myc transformation activity. Cotransfection of low levels of Max expression plasmid stimulated Myc transformation activity, but cotransfection of high levels suppressed it. Mutations in the functionally undefined Max amino- and carboxy-terminal regions outside of the B/HLH/LZ motif partly separated these activities, suggesting various modes of Max regulation. We demonstrate that the Max protein is a nuclear protein in vivo and identify a carboxy-terminal region similar to nuclear localization signals whose integrity is necessary for efficient localization. Two mutants that delete amino- or carboxy-terminal consensus signals for casein kinase II (CKII) exhibited altered gel mobility and DNA-binding potential in vitro and showed modified transforming potential in the Ras cotransformation assay, suggesting that CKII or a CKII-related enzyme may regulate Max function in vivo. Our data suggest that both the ratio of $\mathrm{Myc} / \mathrm{Max}$ hetero-oligomers to Max homo-oligomers and Max-specific regulation can contribute to determining the biological activity of $\mathbf{M y c}$ in vivo.

[Key Words: Myc; Max; cell transformation; nuclear localization]

Received March 31, 1992; revised version accepted September 15, 1992.

The Myc protein plays a crucial but ill-defined role in the proliferative programs of animal cells. Myc is a nuclear phosphoprotein that is induced by mitogenic signals and that is overexpressed in a variety of tumors. Analysis of the amino acid sequence of Myc has revealed structures characteristic of a broad class of sequence-specific DNAbinding proteins that function as dimers or higher multimers (Prendergast and Ziff 1989; Lüscher and Eisenman 1990). These structures include a putative leucine zipper (LZ) and a helix-loop-helix (HLH) motif, both of which serve as protein dimerization interfaces, and a region rich in basic amino acids. The LZ and HLH sequences enable transcription factor proteins to form specific homo- and heterodimers. In such dimers, basic regions contributed by each monomer work together to bind specific DNA sequences (Jones 1990). Functional dimers are formed when the dimerization interface sequences of individual monomers can interact. Thus, the amphipathic helix of the c-Jun protein can interact with itself to form homodimers and heterodimerize with Fos as well. However, the less versatile Fos protein is incapable of homodimerization and must rely on Jun for dimerization.

${ }^{3}$ Corresponding author.
The prediction that Myc can bind DNA specifically was fulfilled initially in two nonphysiological experiments (for review, see Prendergast and Ziff 1992). One was the demonstration that high concentrations of a bacterially expressed c-Myc chimera could bind discrete subsets of sequences in a random oligonucleotide mixture (Blackwell et al. 1990). In another approach it was shown that the basic region sequences of c-Myc could specifically bind DNA when functionally dimerized through the HLH motif of the E12 factor (Prendergast and Ziff 1991b). Work from these and other groups (Halazonetis and Kandil 1991; Kerkhoff et al. 1991) indicated that the sequence specifically contacted by the Myc basic region was CACGTG, a subset of the family of DNA elements recognized by other HLH-containing proteins, called E boxes (Cai and Davis 1990).

The failure to demonstrate sequence-specific DNAbinding by Myc under more physiological circumstances led to the proposal that Myc is incapable of efficient homodimerization and that Myc, like Fos, employs a partner protein to form functional heterodimers. A Mycbinding partner was first identified by screening expression libraries with a c-Myc basic region (B)/HLH/LZ peptide probe, which yielded cDNA clones of a primate protein termed Max (Blackwood and Eisenman 1991). A 
second strategy, the cloning of polymerase chain reaction (PCR)-amplified sequences, which encode peptides homologous to the c-Myc B/HLH, identified Myn, the murine homolog of Max (Prendergast et al. 1991). Max is a 160-amino-acid polypeptide with basic region, $\mathrm{HLH}$, and LZ domains highly homologous to Myc. Unlike Myc, Max has a short amino-terminal domain adjacent to the basic region and a substantial carboxyl-terminal domain that lies beyond the leucine zipper. Max associates avidly with Myc both in vitro (Blackwood and Eisenman 1991; Prendergast et al. 1991) and in vivo (Wenzel et al. 1991; Blackwood et al. 1992) to form heterodimers that bind specifically to the previously defined E-box sequence CACGTG. The identification of Max and the demonstration of the binding specificity of the Myc/Max heterodimer corroborate long-standing hypotheses that Myc functions by forming a complex with specific DNA elements.

In addition to heterodimerization with Myc, Max forms homodimers in vitro that bind in a sequence-specific fashion to the same E-box element recognized by the heterodimer (Prendergast et al. 1991; Berberich and Cole 1992; Kato et al. 1992). Specific DNA binding by both Myc/Max heterodimers and Max homodimers is blocked by CpG methylation within the E-box core sequence CACGTG (Prendergast et al. 1991), suggesting that biological effects of the ciimers may be limited in vivo by DNA methylation. Inspection of the amino acid sequence of Max reveals potential recognition sites for casein kinase II (CKII), and immunoprecipitation from extracts of ${ }^{32} \mathrm{P}$-labeled cells has verified that, like Myc (Lüscher et al. 1989), Max is a phosphoprotein in vivo (Wenzel et al. 1991; Blackwood et al. 1992; R. Hopewell and E.B. Ziff, unpubl.). However, the physiological significance of Myc/Max heterodimers, Max homodimers, and a role for phosphorylation is as yet undefined.

Two lines of evidence suggest that Max facilitates Myc function in vivo by allowing Myc to heterodimerize and specifically bind to DNA. First, it has been demonstrated by coimmunoprecipitation that heterodimers can be detected in vivo (Wenzel et al. 1991; Blackwood et al. 1992). Second, we have reported that Max can augment the ability of Myc to transform rat embryo fibroblasts (REFs) when both are transfected with an activated Ras gene (Prendergast et al. 1991). This effect is consistent with elevation of the level of Myc/Max heterodimers that would represent the biologically active form of Myc.

In the present work, we explore more thoroughly the effects of altering Max levels during Myc/Ras cotransformation. We show that as Max levels are increased, focus formation is first stimulated and then repressed. Mutants of Max within its functionally undefined amino- and carboxy-terminal domains are altered in these capacities in a fashion that parallels changes in the affinities of the mutant heterodimers and homodimers for the E-box-binding site. These results provide direct evidence that Max facilitates Myc biological function by allowing sequence-specific association with DNA. Furthermore, the work establishes that the intracellular ratio of Myc/Max heterodimer to Max homodimer levels contributes to the regulation of Myc biological activity and that the amino- and carboxy-terminal domains of Max have a role in regulating the DNA-binding affinity of dimers containing Max.

\section{Results}

Biphasic effect of Max on Myc/Ras cotransformation of primary REFs

We had observed previously that cotransfection of murine Max augmented the focus formation activity of Myc in a Ras cotransformation assay (Prendergast et al. 1991). In later experiments using a modified transfection protocol that improved the reproducibility of transfection efficiency (see Materials and methods), we found that while this result could be repeated, one could also observe either no effect or a suppressive effect of Max on $\mathrm{Myc} /$ Ras transformation. Therefore, to examine this issue more thoroughly, we titrated the amount of Max against constant Myc and mutant Ras in the REF assay. Tertiary passage REFs were transfected with $5 \mu \mathrm{g}$ of each of the human Myc and mutant Ras expression vectors, along with $0.5,1,2$, or $10 \mu \mathrm{g}$ of the murine Max expression vector cytomegalovirus (CMV) myn (Prendergast et al. 1991), and transformed foci were scored 12-14 days later. We observed that cotransfection of low levels of Max plasmid stimulated Myc transformation activity as observed previously, but high levels suppressed it (Fig. 1). Plotted as a percentage of the number of control foci generated by Myc/Ras alone, the augmentative effect of Max in these experiments was two- to threefold while

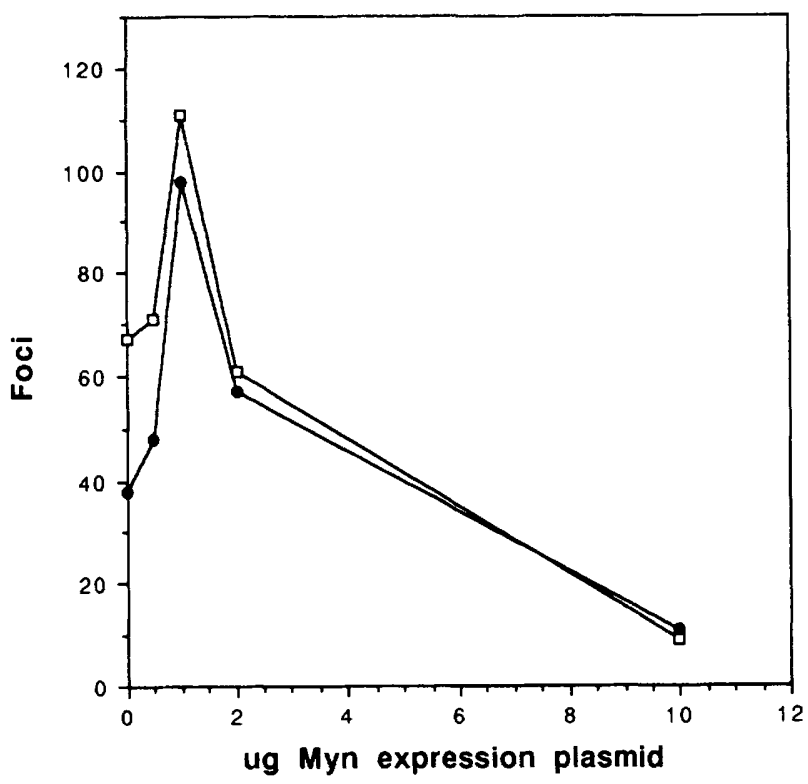

Figure 1. Max expression exerts a biphasic effect on Myc activity in the Ras cotransformation assay. REFs were cotransfected with $5 \mu \mathrm{g}$ of Myc and Ras expression vectors, and the quantity of Myn expression plasmid as noted. (ㅁ) Experiment 1 ; (O) experiment 2. Transformed foci were scored 12-14 days post-transfection (see Materials and methods). 
the suppressive effect was four- to fivefold. The suppression effect produced by the CMV myn expression plasmid could not be explained by promoter competition through vector sequences because transfection of the CMV vector alone had no effect on the efficiency of Myc/Ras foci formation (Table 1). We conclude that Max exhibits a biphasic response for REF cotransformation that is dependent on the relative level of expression plasmid introduced with plasmids encoding Myc and mutant Ras.

Amino- and carboxy-terminal Max mutants separate augmentative and suppressive Max activities in $R E F$ transformation

The biphasic response of Max could be explained by competition of Max for targets of Myc/Max heterodimers that must be bound for transformation to occur. One type of target might be a positive-acting factor or factors specific for Max protein, possibly interacting with the amino- or carboxy-terminal regions that are functionally undefined. If Max overexpression promoted efficient homodimer formation, a second type of target for competition might be a genetic site or sites where Myc/Max heterodimers could exhibit transformationspecific functions. To assess these possibilities and to attempt to separate the augmentative and suppressive effects of Max on Myc transformation activity, we gen- erated a set of mutants in the Max amino- and carboxyterminal regions.

The mutants employed in this study were constructed in the plasmid expression vector CMV myn, encoding a 151-amino-acid peptide, which is the murine form of Max (see Materials and methods; Fig. 2; Prendergast et al. 1991). In vivo, Myn is coexpressed with a 160-aminoacid form generated by splicing and with a 9-amino-acid insert near the amino-terminus (Prendergast et al. 1991). This long form, henceforth called Myn9, was not examined in the present work. The numbering of amino acid residues in wild-type and mutant proteins employed here is based on the sequence of the long form of Myn given in Prendergast et al. (1991). For the purpose of clarity, the wild-type murine Myn protein is referred to as Max. The Myn $\Delta C T$ deletion mutant lacks the entire carboxy-terminal region lying downstream of the $\mathrm{B} / \mathrm{HLH} /$ LZ motif (Prendergast et al. 1991). Myn mutRKK contains a triple point mutation in a carboxy-terminal sequence resembling a nuclear localization signal, replacing amino acids RKK by QAS at amino acids 152154. Two mutants were constructed that disrupt consensus acceptor sites for CKII phosphorylation. Myn $\Delta 5^{\prime} \mathrm{CK}$ contains a double point mutation of two closely spaced sites in the amino-terminus, ${ }^{2} \mathrm{~S}$ to $\mathrm{A}$ and ${ }^{11} \mathrm{~S}$ to $\mathrm{G}$. $M y n \Delta 3^{\prime} \mathrm{CK}$ has a triple point mutation of three sites at amino acids 140, 142, and 144, where all S's are replaced by A's.

Table 1. Titration of wild-type and mutant Max expression plasmids in the Myc/Ras cotransformation assay

\begin{tabular}{|c|c|c|c|c|c|c|c|}
\hline \multirow[b]{2}{*}{ Expression plasmid $(\mu \mathrm{g})$} & \multicolumn{6}{|c|}{ Experiment number ${ }^{a}$} & \multirow[b]{2}{*}{ Mean $^{\mathrm{b}}$} \\
\hline & 1 & 2 & 3 & 4 & 5 & 6 & \\
\hline None & 53 & 21 & 47 & 22 & 28 & 7 & $100 \%$ \\
\hline pcDNAI (vector) $\{10\}$ & - & - & - & - & - & 7 & \\
\hline $\operatorname{Myn}($ w.t. Max) (0.5) & 60 & - & - & 38 & - & 6 & 131 \\
\hline $\operatorname{Myn}(w . t . \operatorname{Max})(1)$ & 98 & - & - & 71 & - & 13 & 225 \\
\hline $\operatorname{Myn}($ w.t. $\operatorname{Max})(2)$ & 59 & - & - & 28 & - & 17 & 116 \\
\hline Myn (w.t. Max) (10) & 10 & - & - & 8 & - & 1 & 24 \\
\hline Myn $\Delta C T(0.5)$ & - & - & 64 & 36 & - & 9 & 145 \\
\hline Myn $\Delta C T(1)$ & - & - & 110 & - & - & 12 & 319 \\
\hline $\operatorname{Myn} \Delta C T(2)$ & - & - & 78 & 35 & - & 11 & 164 \\
\hline $\operatorname{Myn} \Delta \mathrm{CT}(10)$ & - & - & 62 & 16 & - & 12 & 113 \\
\hline Myn mutRKK $(0.5)$ & - & 43 & - & 47 & - & - & 209 \\
\hline Myn mutRKK (1) & - & 29 & - & 47 & - & - & 177 \\
\hline Myn mutRKK (2) & - & 23 & - & 42 & - & - & 153 \\
\hline Myn mutRKK $\mid 10\}$ & - & 14 & - & 55 & - & - & 160 \\
\hline Myn $\Delta 5^{\prime} \mathrm{CK}(0.5)$ & - & - & 95 & - & 47 & - & 189 \\
\hline $\operatorname{Myn} \Delta 5^{\prime} \mathrm{CK}(1)$ & - & - & 131 & - & - & - & 380 \\
\hline Myn $\Delta 5^{\prime} \mathrm{CK}(2)$ & - & - & 26 & - & 23 & - & 65 \\
\hline $\operatorname{Myn} \Delta 5^{\prime} \mathrm{CK}(10)$ & - & - & 7 & - & 6 & - & 17 \\
\hline $\operatorname{Myn} \Delta 3^{\prime} \mathrm{CK}(0.5)$ & - & - & 24 & - & 8 & - & 43 \\
\hline $\operatorname{Myn} \Delta 3^{\prime} \mathrm{CK}\{1\}$ & - & - & 27 & - & 0 & - & 36 \\
\hline $\operatorname{Myn} \Delta 3^{\prime} \mathrm{CK}(2)$ & - & - & 26 & - & 0 & - & 35 \\
\hline $\operatorname{Myn} \Delta 3^{\prime} \mathrm{CK}(10)$ & - & - & 13 & - & 0 & - & 17 \\
\hline
\end{tabular}

Tertiary passage REFs were cotransfected with the Myn (murine Max) expression plasmids in the quantities noted and $5 \mu g$ each of the human c-Myc and mutant H-Ras vectors (see Materials and methods). Transformed foci were scored 12-14 days post-transfection. ${ }^{a}$ Each point noted represents the average of two separate trials.

${ }^{b}$ The mean is depicted as a percentage of the Myc/Ras efficiency (in experiments 1-5), which was set at $100 \%$. The Max titration values were normalized to the $\mathrm{Myc} /$ Ras values within the relevant experiments for each construct. 


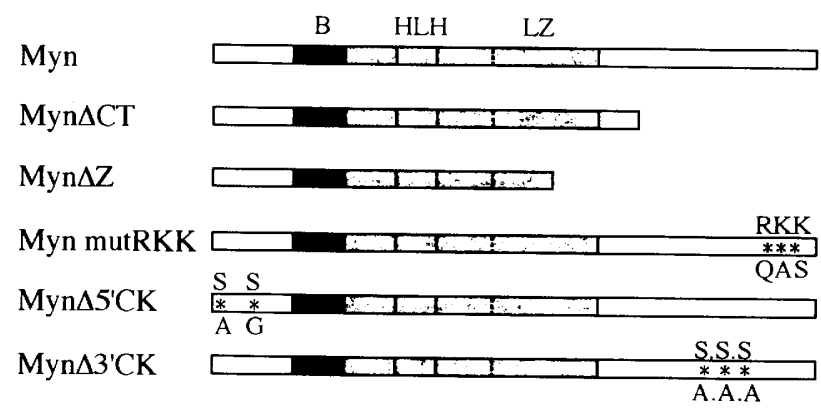

Figure 2. Mutant Max constructions. Graphical representation of mutant Myn proteins generated for this study. Myn $\Delta C T$ sustains a deletion of the entire carboxy-terminal region lying downstream of the $\mathrm{B} / \mathrm{HLH} / \mathrm{LZ}$ motif. $\mathrm{Myn} \Delta \mathrm{Z}$ has a more extensive carboxy-terminal deletion, which encompasses the $\mathrm{LZ}$ motif. Myn mutRKK replaces amino acids ${ }^{143} \mathrm{R}^{144} \mathrm{~K}^{145} \mathrm{~K}$ by QAS. Myn $\Delta 5^{\prime} \mathrm{CK}$ contains a double point mutation of two CKII consensus sites, ${ }^{2} \mathrm{~S}$ to $\mathrm{A}$ and ${ }^{11} \mathrm{~S}$ to $\mathrm{G}$. Myn $\Delta 3^{\prime} \mathrm{CK}$ contains a triple point mutation of three CKII consensus sites, ${ }^{131} \mathrm{~S},{ }^{133} \mathrm{~S}$, and ${ }^{135}$ S all to $\mathrm{A}$.

Each of the Max mutants was titrated as above against constant Myc and mutant Ras in the REF cotransformation assay. The results of the experiments are shown in Table 1 and Figure 3. Myn $\Delta \mathrm{CT}$ and Myn mutRKK augmented Myc cotransformation activity at all ratios of cotransfection. Low levels of $\mathrm{Myn} \Delta 5^{\prime} \mathrm{CK}$ augmented activity somewhat better than wild type. At higher levels, though, this mutant suppressed foci formation slightly more than wild type. Myn $\Delta 3^{\prime} \mathrm{CK}$ lacked the ability to augment Myc cotransformation activity but suppressed focus formation considerably better than wild type. We conclude that amino- and carboxy-terminal Max mutations lying outside of the oligomerization and DNAbinding domains can influence the augmentative and suppressing activities of Max on Myc in Ras cotransformation assays.

\section{Myn mutRKK defines a signal that is necessary for nuclear localization of Max}

All the mutant constructs exhibited altered phenotypes relative to the wild type, suggesting that the stability of the mutant proteins was sufficient to exert the observed biological effects. However, to corroborate the likelihood that mutant proteins stably accumulated in the transformed REF cells, we examined their expression in vitro and in vivo. In vitro-synthesized proteins generated in reticulocyte extracts were observed by SDS-gel electrophoresis (see Fig. 4A). Increased mobility of the $\Delta 3^{\prime} \mathrm{CK}$ mutant relative to the wild-type protein could be the result of reduced phosphorylation by CKII or a similar activity in the extracts (see below). The lower mobility of Myn mutRKK in the gel relative to wild type, despite its lower predicted molecular weight, suggests that the mutation perturbs the carboxy-terminal structure.

To examine the stability of the mutant proteins in vivo, constructs were transiently introduced into COS cells where they could be expressed efficiently owing to the SV40 origin present on the cloning vector. Proteins were monitored 2 days after transfection by indirect immunofluorescence with an anti-murine Max peptide antiserum termed anti-MynCT. This antiserum was raised to a 22-amino-acid carboxy-terminal peptide of the murine Max protein (MynCT) that was coupled to keyhole limpet cyanin (see Materials and methods). From metabolically labeled rodent cells, anti-MynCT immunoprecipitated an $\sim 20-\mathrm{kD}$ polypeptide doublet of similar mobility to in vitro-translated murine Max protein $(R$. Hopewell and E.B. Ziff, unpubl.).

Expression of wild-type murine Max protein gave a strong nuclear stain with anti-MynCT (see Fig. 4B). In addition, we observed nuclear staining of similar intensity for $M y n \Delta 5^{\prime} \mathrm{CK}$ and $\mathrm{Myn} \Delta 3^{\prime} \mathrm{CK}$ (data not shown). Cells transfected with control vector did not produce appreciable fluorescence, indicating that under the conditions employed endogenous proteins could not be detected (data not shown). The stability of the Myn $\Delta C T$ protein could not be examined because it lacked the carboxy-terminal epitope recognized by the antisera. In contrast to the wild-type and other mutant polypeptides, expression of Myn mutRKK produced a general cell stain (see Fig. 4B). Because Myn mutRKK contains a carboxyterminal triple point mutation, we infer that the integrity of this region in Max is necessary for efficient nuclear localization. An alignment of the Max carboxyl terminus with known nuclear localization signals demonstrates sequence similarity to these signals /see Table

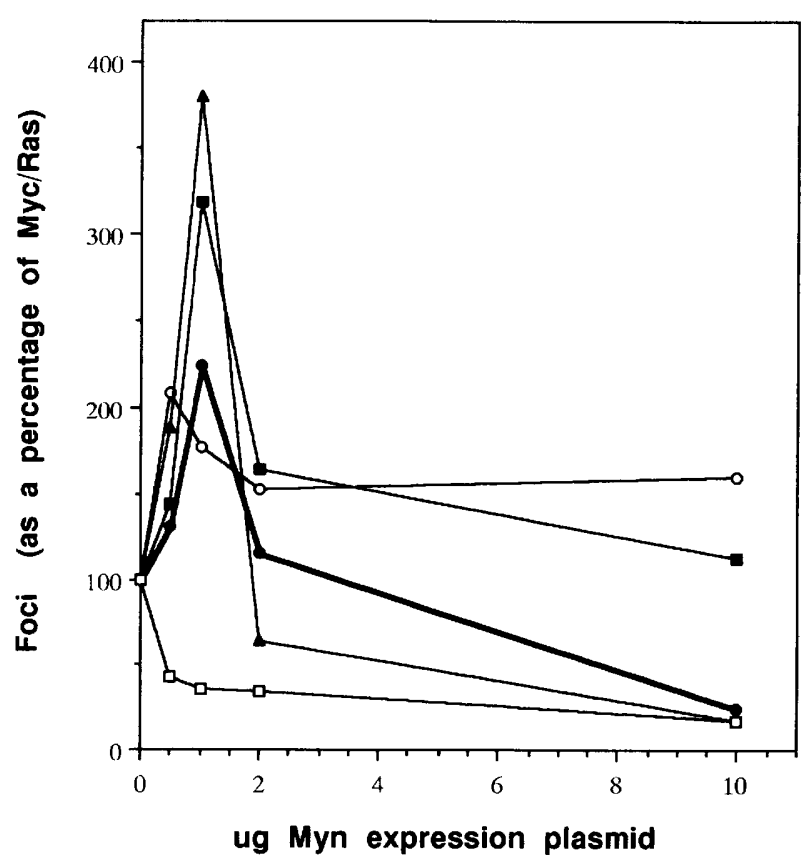

Figure 3. Titration of mutant Max expression plasmids on Myc activity in the Ras cotransformation assay. Each point represents the mean of the data, as shown in Table 1 . Foci values for each construct are expressed as a percentage of the Myc/Ras efficiency, which was set at $100 \%$.(O) Wild-type $\mathrm{Myn}_{\text {; }}$ (O) Myn

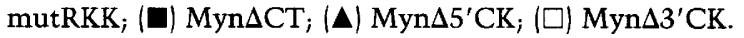




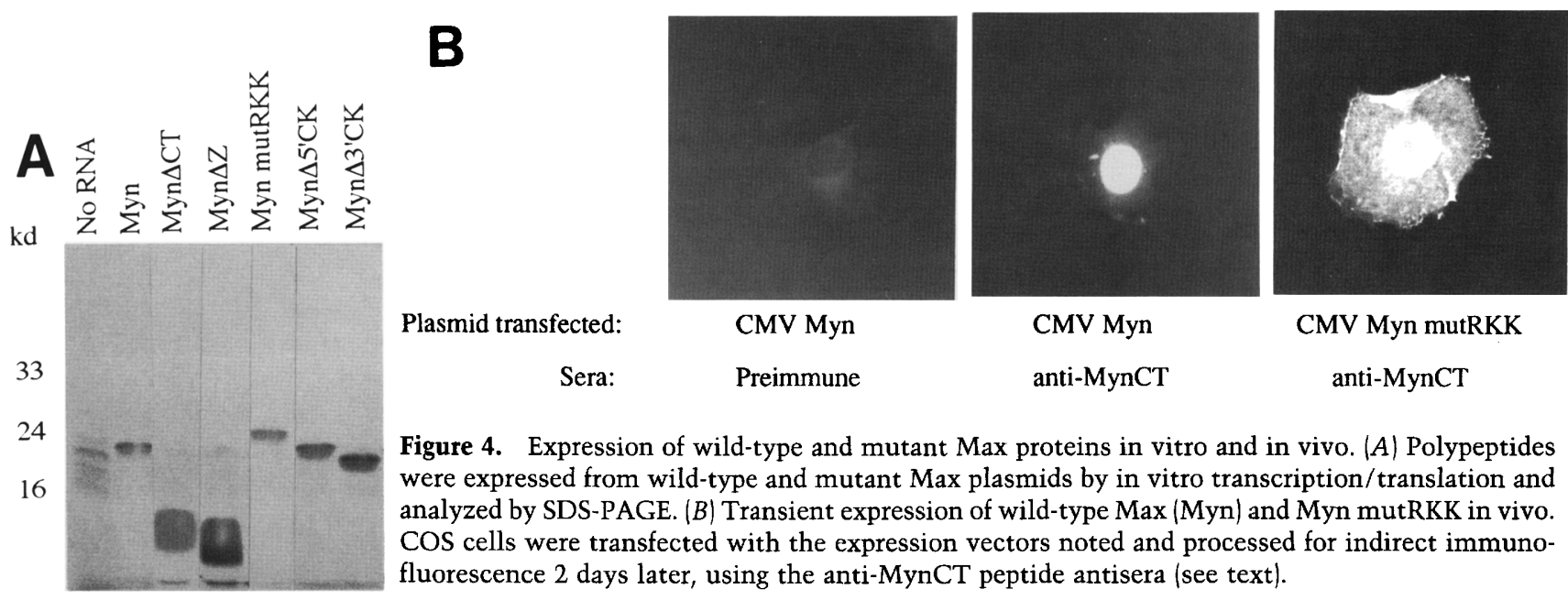

2). We conclude that the murine Max mutants generated for this study are efficiently and stably expressed in vitro and in vivo and that the Max carboxyl terminus encodes a signal responsible for nuclear localization in vivo.

\section{Altered DNA-binding activity of Max mutants lacking CKII consensus sites}

We examined the DNA-binding activity of the in vitrosynthesized mutant Max proteins as a first step toward understanding the means by which their transformation activities might be modified (see Fig. 5A,B). Specific binding of both homodimers and heterodimers to an oligonucleotide containing a Myc recognition site was analyzed by a gel mobility-shift assay.

The CKII consensus site mutants $\mathrm{Myn} \Delta 5^{\prime} \mathrm{CK}$ and $M y n \Delta 3^{\prime} \mathrm{CK}$ displayed altered DNA-binding activity relative to wild-type Max homodimers and Myc/Max heterodimers. As observed previously (Prendergast et al. 1991), weak binding of wild-type Max homodimers could be detected above a background binding activity present in reticulocyte extract. The low binding activity could be clearly discerned by comparison to a negative control mutant, MynZ, which lacks the LZ motif and cannot oligomerize (Prendergast et al. 1991). The background activity was not inhibited by anti-MynCT peptide an-

Table 2. Nuclear localization signals

\begin{tabular}{|c|c|c|}
\hline Protein & Sequence & Reference \\
\hline $\operatorname{Max}$ & $\mathrm{P}^{149} \mathrm{Q}$ S RKKLRM & this work \\
\hline c-Myc & $\mathrm{P}^{320} \mathrm{AAKRLKVD}$ & $\begin{array}{c}\text { Dang and Lee (1988), } \\
\text { Stone et al. (1987) }\end{array}$ \\
\hline N-myc & $\mathrm{P}^{337} \mathrm{PQKK}$ I K S & Dang and Lee (1989) \\
\hline c-Myb & $\mathrm{P}^{521}$ L LKK I KQ & Dang and Lee (1989) \\
\hline p53 & $\mathrm{P}^{316} \mathrm{QPKKKP}$ & Dang and Lee (1989) \\
\hline SV40 large $T$ & $\mathrm{P}^{126} \mathrm{KKKRKVE}$ & $\begin{array}{l}\text { Dingwall and Laskey } \\
\text { (1986) }\end{array}$ \\
\hline
\end{tabular}

Comparison of the Max carboxy-terminal region with selected nuclear localization signals containing an amino-terminal helix-breaking proline (Dang and Lee 1989). tiserum (data not shown) even though its similar mobility suggested that it may be the result of endogenous Max in the reticulocyte extract. Myn mutRKK exhibited weak binding activity similar to wild-type Max. As observed previously (Prendergast et al. 1991), Myn $\Delta$ CT bound the CACGTG recognition site oligonucleotide more strongly than wild-type Max and produced a rela-

$\mathbf{A}$

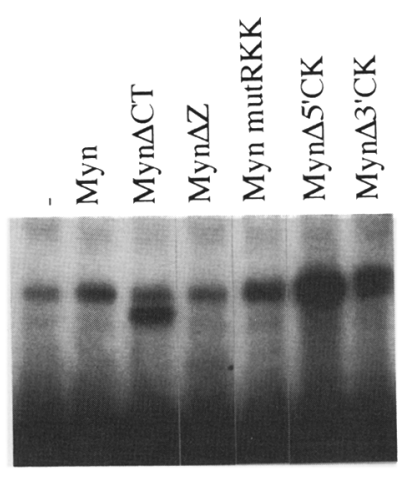

B

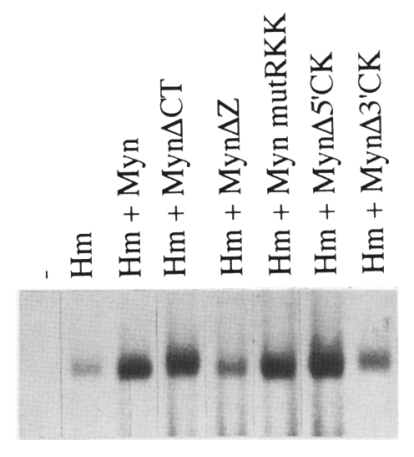

Figure 5. DNA binding by Max mutants. A gel mobility-shift assay was used to monitor specific DNA binding of wild-type and mutant Max proteins synthesized in vitro. The oligonucleotide probe employed in the binding reaction contained the specific recognition sequence GACCACGTGGTC (Halazonetis and Kandil 1991; Prendergast et al. 1991). (A) Specific DNA binding by Max homodimers; $(B)$ specific DNA binding by Myc/ Max heterodimers. 
tively more rapid mobility shift consistent with its smaller size. Both the CKII consensus site mutants also showed an increase in specific DNA binding compared with Max. Myn $\Delta 3^{\prime} \mathrm{CK}$ bound the CACGTG recognition site oligonucleotide two- to threefold better than wild type, producing a slightly more retarded shift band. Similarly, $\operatorname{Myn} \Delta 5^{\prime} \mathrm{CK}$ specifically bound DNA at least 10 fold better as a homodimer than wild-type Max. Both CKII mutants also showed changes in DNA binding as heterodimers with Myc. A subtle but detectable increase of $\mathrm{Myc} / \mathrm{Myn} \Delta 5^{\prime} \mathrm{CK}$ binding to the CACGTG recognition site nucleotide relative to wild-type Myc/Max was observed. In contrast, $\mathrm{Myc} / \mathrm{Myn} \Delta 3^{\prime} \mathrm{CK}$ was observed to specifically recognize DNA two- to threefold more poorly than wild-type Myc/Max.

The observed changes in DNA binding by homodimers and heterodimers could not be attributed to variations in the amount of mutant proteins added to the DNA-binding reactions, because all were synthesized and added at similar levels (Fig. 4A; data not shown). We tested the possibility that the variations were the result of changes in dimerization efficiency in the following experiments. Heterodimerization of in vitro-translated Max mutants with c-Myc was examined by coimmunoprecipitation with anti-Myc antisera (Fig. 6A). The mutants were coprecipitated with an efficiency similar to wild type Max, with the exception of Myn $\Delta Z$, which lacks a LZ motif
(Prendergast et al. 1991). The ability of the Max mutants to homodimerize was analyzed by their ability to bind to Myn $\Delta$ C-Sepharose (generously provided by A. FerréD'Amare and S. Burley, Rockefeller University, New York), an affinity matrix containing a bacterially produced version of Myn $\Delta C T$ (Prendergast et al. 1991). Myn $\Delta \mathrm{C}$ lacks the Max carboxyl terminus but encodes the complete B/HLH/LZ motif. We observed no difference in the efficiency of Myn $\Delta \mathrm{C}-$ Sepharose binding between wild-type Max and the mutant Max proteins with altered DNA-binding activity (Fig. 6B). We conclude that the observed changes in the DNA-binding activity of the Max mutants cannot be accounted for by changes in their homo- or heterodimerization efficiency.

To establish that the in vitro-encoded Max employed in these experiments is phosphorylated and that the mutations in Myn $\Delta 5^{\prime} \mathrm{CK}$ and Myn $\Delta 3^{\prime} \mathrm{CK}$ altered the level of phosphorylation, we analyzed tryptic peptide maps of these products synthesized and phosphorylated in reticulocyte lysates. The map of the wild-type protein gave a complex pattern of phosphopeptides shown in Figure 7B, which was consistent with the presence of five or more potential CKII phosphorylation sites within the Max protein. The map of the mutant Myn $\Delta 3^{\prime} \mathrm{CK}$ shown in Figure $7 \mathrm{D}$ gave a greatly simplified pattern containing two prominent spots and lacking those spots found in the wild-type map, which migrated near the origin of the
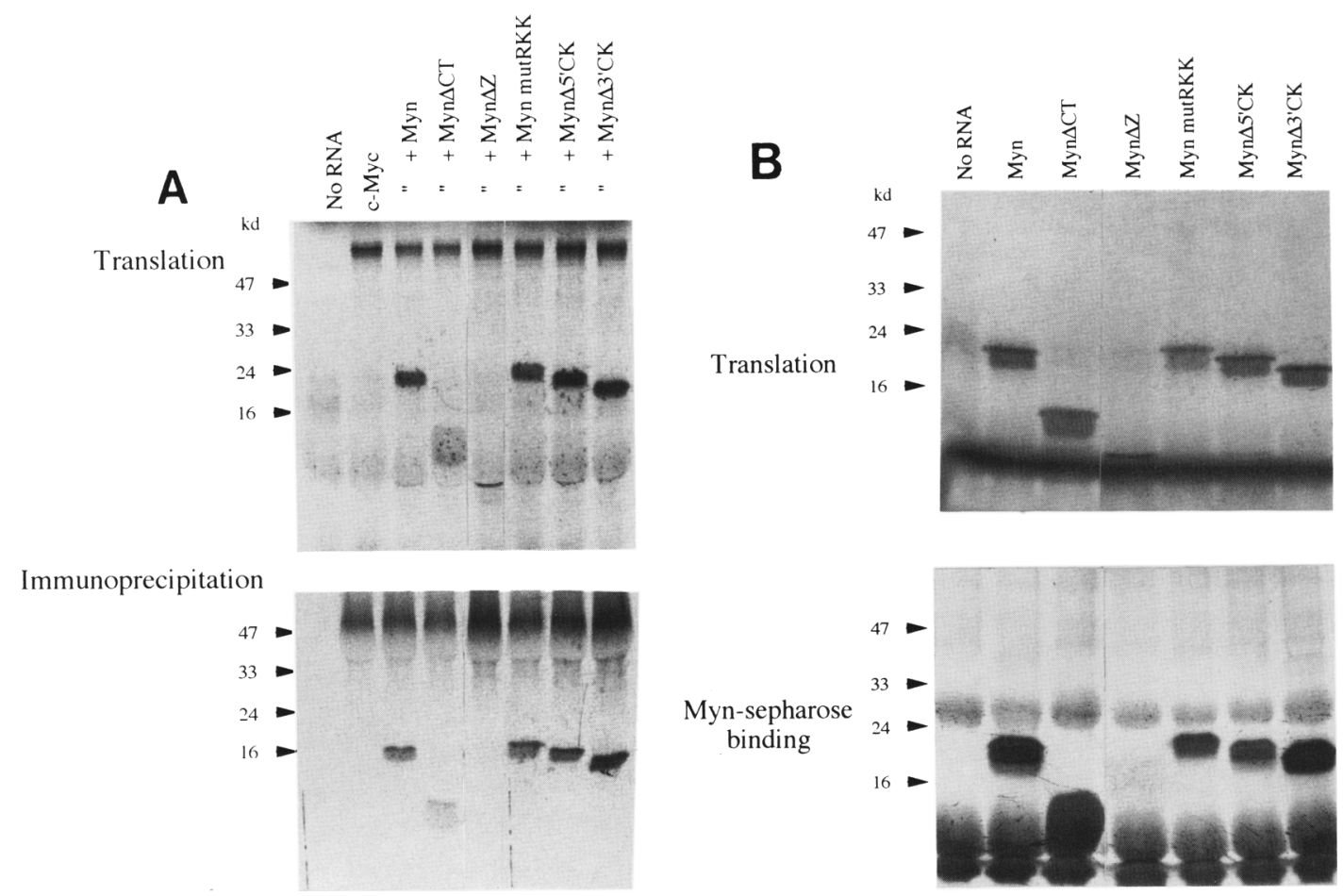

Figure 6. Dimerization by Max mutants. $(A)$ Heterodimerization. Wild-type Max and the Max mutants were cotranslated with c-Myc and subjected to immunoprecipitation with anti-Myc antisera as described previously (Prendergast et al. 1991). (Top) SDS-PAGE of ${ }^{35} \mathrm{~S}$-labeled proteins generated by in vitro translation; (bottom) SDS-PAGE of immunoprecipitated proteins. $(B)$ Homodimerization. Wild-type Max and Max mutants were translated in vitro and mixed with Myn-Sepharose beads (provided by A. Ferré-D'Amare and S. Burley). Following incubation, the beads were washed three times in binding buffer. Bound proteins were eluted by boiling in SDS gel buffer and analyzed by SDS-PAGE. (Top) SDS-PAGE of ${ }^{35}$ S-labeled proteins generated by in vitro translation; (bottom) SDS-PAGE of proteins bound to Myn-Sepharose. 
fractionation. The latter products migrated in the position anticipated for multiply phosphorylated peptides such as the tryptic peptide from the Max carboxyl terminus, a peptide that is predicted to contain three or more CKII sites. The two prominent spots of Figure 7D were absent in the map of Myn $\Delta 5^{\prime} \mathrm{CK}$ shown in Figure 7C, which, however, contained the low mobility, highly phosphorylated products assigned above to the carboxyl terminus. We conclude that the prominent spots of Myn $\Delta 3^{\prime} \mathrm{CK}$ in Figure 7D, also seen in the wild-type map in Figure $7 \mathrm{~B}$ but absent from the $\mathrm{Myn} \Delta 5^{\prime} \mathrm{CK}$ map of Figure $7 \mathrm{C}$, are derived from the amino-terminal CKII sites and represent the singly and doubly phosphorylated forms of the amino-terminal Max tryptic peptide. These results show that the amino- and carboxy-terminal CKII site mutations block specific phosphorylation of Max introduced in the reticulocyte lysates.

Taken together, the data suggest that CKII or a CKIIlike activity present in reticulocyte extracts regulates Max DNA-binding activity. Furthermore, the data indicate that mutations of the CKII consensus signals in the amino- and carboxy-terminal regions of Max alter the DNA-binding capabilities of Max in both homotypic and heterotypic forms.

\section{Discussion}

\section{Biological effects of Max expression on Myc function}

The major conclusion of our work is that Max can facilitate or suppress Myc function in vivo in a manner that is dependent on both the ratio of Myc/Max heterodimers to Max homodimers and on Max-specific regulation. Varying the transfection ratio of Max to Myc expression vector in the Ras cooperation assay exerted a biphasic effect on the transformation activity of c-Myc: Cotransfection of low levels of Max expression plasmid consistently stimulated Myc transformation activity, but cotransfection of high levels suppressed it. The biological activity of Max mutants indicated that Max regulation in its amino- and carboxy-terminal regions was also important in controlling Myc function in the cotransformation assay.

We interpret the results of our titration experiments to mean (1) that the intracellular ratio of Myc to Max protein levels plays an important role in establishing the biological activity of Myc in vivo, and (2) that Myc/Max heterodimers activate while Max/Max homodimers repress. This interpretation is consistent with the current picture of oligomerization potentials in vitro and in vivo. From in vitro studies, several groups have offered evidence for the existence of Myc homo-oligomers (Dang et al. 1989; Blackwell et al. 1990; Halazonetis and Kandil 1991; Kerkhoff et al. 1991; Prendergast and Ziff 1991b; Prendergast et al. 1991). However, interpretation of this work is complicated by the possible presence of Mycbinding partner proteins in translation lysates or the use of various means to drive efficient oligomerization and DNA binding that may not be physiologically relevant. Furthermore, a direct test for in vivo homo-oligomerization of the Myc B/HLH/LZ region has failed to detect any interaction (Dang et al. 1991). The in vivo existence
A

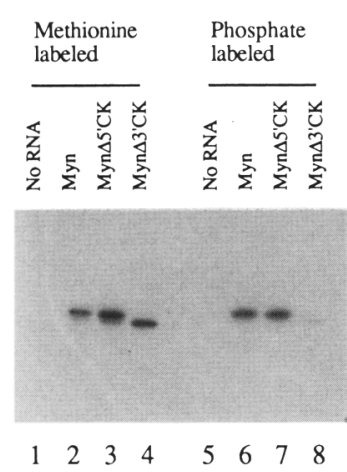

$\mathrm{C}$

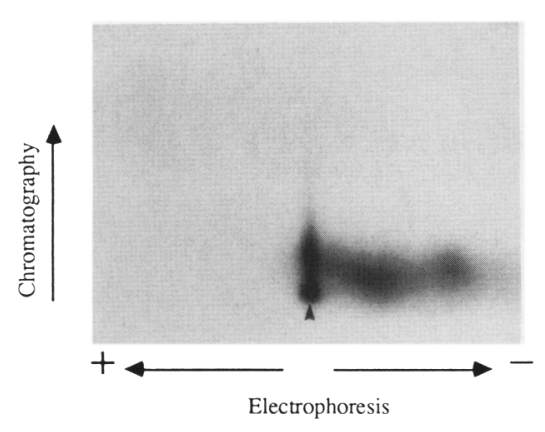

B

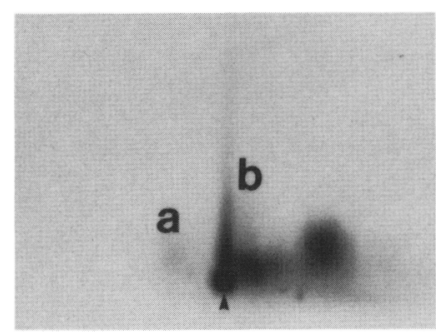

$\mathrm{D}$

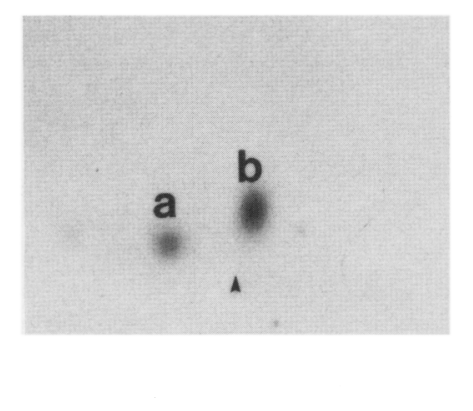

Figure 7. In vitro phosphorylation state of CKII consensus site Max mutants. $(A)$ Wild-type and mutant Myn proteins were translated in reticulocyte lysate with either $\left[{ }^{35}\right.$ S $]$ methionine (lanes 1-4) or [ ${ }^{32}$ P]ATP (lanes 5-8). Labeled protein was then immunoprecipitated using anti-MynCT peptide antiserum and separated on a $15 \%$ polyacrylamide gel. (B) Phosphopeptide map of wild-type Max protein. ${ }^{32}$ P-Labeled Max was excised from the gel described in $A$, digested with trypsin, and separated in the first dimension by electrophoresis and in the second dimension by chromatography. The origin is marked with an arrowhead. Spots marked a and $\mathrm{b}$ are interpreted to be di- and monophosphorylated forms, respectively, of an amino-terminal peptide mutated in Myn $\Delta 5^{\prime} \mathrm{CK}$, discussed in text. $(C)$ As in $B$, using Myn $\Delta 5^{\prime} C K$. $(D)$ As in $B$, using $M y n \Delta 3^{\prime} \mathrm{CK}$. 
of a homotypic form of Myc is therefore in question. In contrast, the existence of Myc/Max hetero-oligomers in vivo has been demonstrated (Wenzel et al. 1991; Blackwood et al. 1992).

In the standard Myc/Ras cooperation assay in primary REFs, Myc levels are elevated through enforced expression from a strong constitutive promoter and Max protein is expressed only from the endogenous gene. The ratio of Myc to Max in these cells should therefore be elevated relative to an untransfected cell. In the presence of excess exogenous Myc, the endogenous Max protein would be saturated as a heterodimer. Because Myc homodimerizes poorly (Smith et al. 1990; Dang et al. 1991), if at all, any excess Myc would not contribute appreciably to additional dimer formation. Thus, under the standard conditions of the Myc/Ras assay, the level of Max would be the limiting factor in generating a heterodimer. If Max levels are increased through cotransfection of a Max expression vector, excess Myc can be recruited into heterodimers. However, as the Max levels surpass Myc, Max will have the opportunity to homodimerize and any effects of Max homodimers will be manifested. Therefore, as Max levels increase, the biphasic nature of the titration first reflects the increase in the heterodimer level and later the production of a homodimer.

\section{Biological effects of amino- and carboxy-terminal Max mutants}

The biphasic response generated by a set of Max mutants in the functionally undefined amino- and carboxy-terminal regions supported the above interpretation that Myc function in vivo is affected by the intracellular ratio of Myc/Max heterodimers to Max homodimers. More importantly, however, the changes in the shapes of the mutant titration curves relative to wild type indicated that Max can also influence Myc biological activity through means other than dimerization. We have concluded that there are qualitative differences in the manner in which the various mutants function in vivo. Our analyses were not extensive enough to warrant quantitative conclusions regarding the effectiveness of augmentation or suppression of Myc cotransformation by the mutant Max proteins. Further characterization of the in vivo expression is required to address this. However, several types of mutants were informative, allowing separation of the focus-activating and -suppressing activities of Max in the Ras cotransformation assay, identification of a putative nuclear localization signal, and a possible role for CKII in regulating Max in vivo.

We and others (Blackwood et al. 1992; Mäkelä et al. 1992) have shown that the Max protein is nuclear localized in vivo, and in this work we identified a carboxyterminal region related to nuclear localization signals that is necessary for proper localization. Another group has shown that this region of Max is sufficient for nuclear localization of a heterologous protein (Kato et al. 1992). The two mutants that lack the nuclear localization signal, Myn mutRKK and Myn $\Delta \mathrm{CT}$, appeared to be impaired for suppression of $\mathrm{Myc} / \mathrm{Ras}$ foci at higher ratios of transfection. The lack of a functional nuclear localization signal in each of these proteins suggests the interpretation that neither Myn $\Delta \mathrm{CT}$ nor Myn mutRKK localize efficiently in the nucleus as homodimers. However, as heterodimers they should be localized as well as wild type, owing to the functional Myc signal. The net effect of their expression (relative to wild-type Max) would therefore be a relative increase in the nuclear level of heterodimers. Alternatively, because they lack the nuclear localization signal, Myn $\Delta C T$ and Myn mutRKK might not achieve levels sufficiently high to repress focus formation.

\section{Max amino-terminal regulation and a possible role for CKII}

The amino-terminal CKII mutant $\mathrm{Myn} \Delta 5^{\prime} \mathrm{CK}$ was similar to wild-type Max in its ability to augment and suppress transformation but had a somewhat more pronounced biphasic effect. This effect correlated with its in vitro DNA-binding activity, as Myn $\Delta 5^{\prime} \mathrm{CK}$ was observed to bind DNA better as both a homo- and heterodimer. It is therefore possible to interpret its more pronounced phenotype relative to wild-type Max as due first to its increased DNA-binding affinity as a heterodimer and then as a homodimer, as it is titered into the assay. The mutations that change the transformation activity and in vitro DNA-binding potential of the Myn $\Delta 5^{\prime} \mathrm{CK} \mathrm{mu-}$ tant block phosphorylation at CKII sites adjacent to the basic region. Effects of phosphorylation adjacent to the basic region have been reported by other groups. First, Max expressed in Escherichia coli (Berberich and Cole 1992; Kato et al. 1992) binds DNA more avidly than that synthesized by in vitro translation (Prendergast et al. 1991), suggesting that modification can affect Max DNA-binding activity. More importantly, it has recently been demonstrated that phosphorylation of the Max amino-terminal region by CKII in vitro can suppress the DNA-binding activity of Max homodimers (Berberich and Cole 1992). Taken together, the data suggest that CKII may act in vivo to regulate Max function through control of DNA binding by homo- and heterodimers.

Similar to Max, a common feature of B/HLH and basic leucine zipper (b-ZIP) proteins is the presence of a CKII site immediately upstream of the basic region, for example, Jun (Boyle et al. 1991), Fos (G.C. Prendergast and E.B. Ziff, unpubl.), and Myc (Lüscher et al. 1989). In the case of Jun, phosphorylation immediately upstream of the basic region has been implicated in regulation of Jun DNA binding in vivo (Boyle et al. 1991). In Myc, the possibility that the CKII site upstream of the basic region is important for regulation is suggested by its mutation in the v-Myc protein encoded by the avian retrovirus MC29 (G.C. Prendergast and E.B. Ziff, unpubl.). We have demonstrated that the amino-terminal CKII mutant Myn $\Delta 5^{\prime} C K$ bound DNA significantly better than wildtype Max. This suggests that DNA binding by Max and other b-ZIP and bHLH proteins in vivo might be controlled by phosphorylation adjacent to the basic region. In the simplest model, phosphorylation might act 
through electrostatic repulsion between phosphate residues on the protein and DNA backbone to "gate" access of dimers to their DNA recognition sequences. However, other mechanisms could also limit DNA binding, for example, interaction between the negatively charged phosphate residues at the CKII sites and the positively charged amino acids in the basic region.

\section{Max carboxy-terminal function and effects of CKII}

In contrast to the amino-terminal mutant, the carboxyterminal CKII mutant Myn $\Delta 3^{\prime} \mathrm{CK}$ was observed to effectively suppress, but not augment, Myc cotransformation activity. The effect of carboxy-terminal phosphorylation of Max on in vitro DNA binding of Max homo- and heterodimers offers a possible explanation of the in vivo result; in the mutant dephosphorylated state, Max homodimers bind DNA more efficiently, whereas Max/ Myc heterodimers bind DNA less efficiently. Thus, carboxy-terminal dephosphorylation of Max could block Myc function by favoring Max homodimer binding to DNA over Myc/Max heterodimers. The mechanism by which carboxy-terminal phosphorylation affects DNA binding by Max homo- and heterodimers is unclear. The change in DNA-binding activity of Myn $\Delta 3^{\prime} \mathrm{CK}$ could not be explained by an alteration in heterodimer formation, because the mutant protein coimmunoprecipitated with an efficiency indistinguishable from wild-type Max. Furthermore, no change in homodimerization efficiency of Myn $\Delta 3^{\prime} \mathrm{CK}$ relative to wild-type Max could be detected, as assayed by binding to Myn $\Delta \mathrm{C}-$ Sepharose beads.

The proximity of the carboxy-terminal cluster of CKII sites to the nuclear localization signal in Max suggests a possible effect of phosphorylation on nuclear localization in addition to the effect on DNA binding. In T antigen, phosphorylation at adjacent sites by CKII enhances (Rihs et al. 1991) and, by p34 ${ }^{\text {cdc2 }}$, impairs (Jans et al. 1991) the efficiency of nuclear localization. However, Myn $\Delta 3^{\prime} \mathrm{CK}$ is able to localize in the nucleus (G.C. Prendergast, unpubl.), which suggests that carboxy-terminal phosphorylation does not have a major role in the regulation of Max nuclear localization. It is possible that the repression of focus formation by $M y n \Delta 3^{\prime} \mathrm{CK}$ is derived from some other biological function of the Max carboxyl terminus apart from DNA binding or nuclear localization, which affects Myc-dependent activity and is altered by phosphorylation.

One prediction that arises from our work is the potential that genetic alterations of the Max carboxyl terminus might be observed in human tumors. We have shown that Myn $\Delta C T$, a Max carboxy-terminal deletion mutant, and Myn mutRKK, a nuclear localization signal mutant, both activate Myc transformation activity more efficiently than wild-type Max. Consistent with our findings, others have shown recently that a naturally occuring carboxy-terminal truncated form of Max of 103 amino acids (termed $\triangle \mathrm{Max}$ ) also augments the cotransformation activity of Myc (Mäkelä et al. 1992). On the basis of these data, one could speculate that mutations resulting in carboxy-terminal truncation or mutation of
Max could contribute a step in tumorigenesis by indirectly controlling Myc activity.

In summary, the results presented here provide evidence that Max homodimers can exert a regulatory role in which they block Myc-dependent functions associated with Ras cooperation. The mechanism may depend on competition between Max homodimers and $\mathrm{Myc} /$ Max heterodimers for DNA-binding sites. Because mutations in Max, which alter its potential for amino- and carboxy-terminal Max phosphorylation, change the repressive activity of the homodimer, changes in the phosphorylation state of Max in vivo could potentially alter its activity as an antagonist of Myc action. Three groups have shown that Max can be metabolically labeled with ${ }^{32} \mathrm{PO}_{4}$ (Wenzel et al. 1991; Blackwood et al. 1992; R. Hopewell and E.B. Ziff, unpubl.), but regulation of this phosphorylation has not yet been reported. A test of this possibility will require in vivo analysis of changes in Max phosphorylation that are concomitant with regulation of Myc activity.

\section{Materials and methods}

\section{Plasmid constructions}

All mutants were generated by standard PCR techniques with oligonucleotide primers (Genosys, Houston TX) containing the desired mutations. The target for all amplifications was a myn cDNA encoding the 151-amino-acid form of the Myn protein (Prendergast et al. 1991). All PCR products were cloned as HindIII-EcoRI fragments into pcDNAI (InVitrogen), which allows for expression in vitro by the $\mathrm{T} 7$ promoter and in vivo by the CMV enhancer/promoter. The mutations were verified by DNA sequencing. The carboxy-terminal deletions Myn $\Delta C T$ (that contains an intact $\mathrm{B} / \mathrm{HLH} / \mathrm{LZ}$ motif) and $\mathrm{Myn} \Delta \mathrm{Z}$ (which lacks a complete LZ motif) have been described (Prendergast et al. 1991). Myn mutRKK replaces amino acids ${ }^{143} \mathrm{R}^{144} \mathrm{~K}^{145} \mathrm{~K}$ by QAS; Myn $\Delta 5^{\prime} \mathrm{CK}$ contains a double point mutation of two CKII consensus sites, ${ }^{2} \mathrm{~S}$ to $\mathrm{A}$ and ${ }^{11} \mathrm{~S}$ to $\mathrm{G}$; $\mathrm{Myn} \Delta 3^{\prime} \mathrm{CK}$ contains a triple point mutation of three CKII consensus sites, ${ }^{131} \mathrm{~S},{ }^{133} \mathrm{~S}$, and ${ }^{135} \mathrm{~S}$ all to A. Myn mutRKK was generated in two steps by first amplifying the amino-terminal fragment with the $5^{\prime}$ primer mbh2-5' (Prendergast et al. 1991) and the mutant 3' primer $\left(5^{\prime}\right)$-CTCCATCCGGAGACTCGCCTGGCTCTGGG and the carboxy-terminal fragment with the mutant $5^{\prime}$ primer $\left(5^{\prime}\right)$ CCCAGAGCCAGGCGAGTCTCCGGATGGAG and a 3' SP6 sequence primer (Promega), and then generating the full mutant by mixing the two reactions and amplifying with $\mathrm{mbh} 2 / 5^{\prime}$ and SP6 primers. Myn $\Delta 3^{\prime} \mathrm{CK}$ was constructed in a similar fashion, except that the primers were (antisense) (5')-CTTCAGGCTCGGCTTCTGCAGCGGCGTCTGAACCC and (sense) (5')-GGGTTCAGACGCCGCTGCAGAAGCCGAGCCTGAAG. Myn $\Delta 5^{\prime} \mathrm{CK}$ was generated using the $5^{\prime}$ primer $\left(5^{\prime}\right)$-AGAAGCTTGGAAATGGCCGATAACATGACATCGAGGTGGAGGGCGACG and the 3' SP6 sequencing primer.

\section{Preparation of anti-MynCT peptide antisera}

A 22-mer peptide (MynCT) containing the carboxy-terminal Myn sequence LQTNYPSSDNSLYTNADGGTIS (Prendergast et al. 1991) was synthesized, conjugated to keyhole limpet cyanin (Cambridge Research Biochemicals, Wilmington, DE), and used for production of rabbit antisera (Pocono Rabbit Farm, Ca- 
nadensis, PA). Crude antiserum was used for immunofluorescence and immunoprecipitation experiments.

\section{Indirect immunofluorescence of transiently transfected COS cells}

Cells were seeded onto glass coverslips in Dulbecco modified Eagle medium (DMEM) containing 10\% FBS. The following day they were transfected using a modified calcium phosphate precipitation protocol (Chen and Okayama 1987) with $20 \mu \mathrm{g}$ of wild-type or mutant Myn plasmid DNAs. Following incubation overnight at $37^{\circ} \mathrm{C}$ in $3-4 \% \mathrm{CO}_{2}$, cultures were washed and refed. Coverslips were processed $48 \mathrm{hr}$ later for indirect immunofluorescence as described (Prendergast and Ziff 1991a).

\section{Immunoprecipitations}

Ten microliters of ${ }^{35} \mathrm{~S}$-labeled c-Myc and Max proteins generated by in vitro translation were subjected to immunoprecipitation with anti-Myc or anti-Myn CT peptide antisera and analyzed as described previously (Prendergast and Ziff 1991a).

\section{Binding of wild-type and mutant Max proteins to Myn-Sepharose}

The affinity matrix Myn $\Delta \mathrm{C}-$ Sepharose was generated and provided by A. Ferré-D'Amare and S. Burley (Rockefeller University, New York). Briefly, Myn $\Delta C T$ (Prendergast et al. 1991) was expressed in $E$. coli by an expression plasmid that adds an amino-terminal extension containing 6 consecutive histidine residues. The extension allowed purification of the bacterial fusion protein, termed Myn $\Delta \mathrm{C}$, by nickel Sepharose chromatography (Pharmacia). For the binding experiments, the coupled affinity matrix was generated by mixing $0.5 \mathrm{ml}$ of nickel-Sepharose (Pharmacia) with $50 \mu \mathrm{g}$ of purified Myn $\Delta \mathrm{C}$ in a buffer containing $10 \%$ glycerol, $1 \mathrm{M} \mathrm{KCl}, 20 \mathrm{~mm}$ HEPES (pH 8.4), $5 \mathrm{~mm}$ imidazole, and $1 \mathrm{mM}$ PMSF. Binding was performed by mixing $25 \mu \mathrm{l}$ each of ${ }^{35} \mathrm{~S}$-labeled Max and Max mutant proteins (synthesized in vitro), Myn $\Delta \mathrm{C}-$ Sepharose, and $3 \times$ buffer A (150 mM $\mathrm{NaCl}, 30 \mathrm{~mm}$ Tris- $\mathrm{Cl}$ at $\mathrm{pH} 7.5$ ). Following incubation for 30 min at $37^{\circ} \mathrm{C}$, the beads were washed twice with $250 \mu \mathrm{l}$ of $1 \times$ buffer $\mathrm{A}$ and twice in $200 \mathrm{~mm} \mathrm{NaCl}, 10 \mathrm{~mm}$ Tris-Cl (pH7.5). Bound proteins were eluted by boiling the Myn-Sepharose beads in SDS gel buffer and analyzed by SDS-PAGE and fluorography.

\section{DNA-binding assays}

DNA-binding assays were carried out essentially as described (Prendergast et al. 1991), using $\sim 1$ ng of a specific oligonucleotide probe containing a Myc recognition site GACCACGTGGTC (Halazonetis and Kandil 1991). Protein-DNA complexes were fractionated on $4 \%$ or $8 \%$ polyacrylamide gels $\{37.5: 1$ acrylamide/bisacrylamide) in $1 \times \mathrm{TBE}$, fixed, and processed for autoradiography.

\section{Phosphopeptide mapping of wild-type and mutant Max proteins}

Plasmids pCMVMyn, CMVMyn $\Delta 5^{\prime} \mathrm{CK}$, and CMVMyn $\Delta 3^{\prime} \mathrm{CK}$ were transcribed in vitro and translated in reticulocyte lysate containing complete amino acids and $100 \mu \mathrm{Ci}$ of $\left[\gamma^{32} \mathrm{P}\right] \mathrm{ATP}$. Wild-type and mutant Max proteins were then immunoprecip. itated with anti-MynCT peptide antisera and washed as described (Prendergast et al. 1991). Immunoprecipitated protein was then separated on a $12 \%$ polyacrylamide gel, visualized by autoradiography, and excised from the gel. Samples were processed for phosphopeptide mapping (Hunter and Sefton 1980). The protein was eluted from the gel by boiling for $5 \mathrm{~min}$ in 4 $\mathrm{mg} / \mathrm{ml}$ of ammonium bicarbonate containing $5 \%$ mercaptoethanol and $0.1 \%$ SDS and incubated overnight at $37^{\circ} \mathrm{C}$. The protein was precipitated with TCA, washed three times with acetone, and lyophilized. The protein was then oxidized with perfomic acid (formic acid $/ \mathrm{H}_{2} \mathrm{O}_{2}, 9: 1$ ) for $1 \mathrm{hr}$ at $0^{\circ} \mathrm{C}$, washed and treated with trypsin (Boehringer Mannheim; HPLC Pure) overnight at $37^{\circ} \mathrm{C}$. The sample was lyophilized in $\mathrm{H}_{2} \mathrm{O}$ three times, and spotted onto Whatman cellulose thin layer chromatography plates and electrophoresed for $30 \mathrm{~min}$ at $1.5 \mathrm{kV}$ in buffer containing $2.5 \%$ formic acid and $7.8 \%$ acetic acid, and chromatographed in the second dimension in buffer containing $37.5 \%$ butanol, $25 \%$ pyridine, and $7.5 \%$ acetic acid. The dried plates were autoradiographed for 3 days.

\section{REF transformation assay}

Five T175 flasks of primary REFs received from the vendor (Whittaker Bioproducts) were allowed to recover for $24 \mathrm{hr}$ at $37^{\circ} \mathrm{C}$ and were then split into twenty $15-\mathrm{cm}$ dishes. Cells were cultured to $\sim 50 \%$ confluence, harvested, and divided into 40 freezer vials for storage in liquid nitrogen. For transfection, tertiary passage fibroblasts were prepared by culturing one vial of frozen cells into eight $10-\mathrm{cm}$ dishes. Transfections were carried out in duplicate 3 days after culturing from frozen storage $\left(\sim 2 \times 10^{5}\right.$ fibroblasts per dish). Five micrograms of the Myc (Kelekar and Cole 1987) and mutant H-Ras (Land et al. 1983) plasmids and $0-10 \mu \mathrm{g}$ of the wild-type or mutant CMV Myn plasmids were used for transfections, with the pBluescript SK + vector (Stratagene) used as carrier DNA to bring the total plasmid DNA to $20 \mu \mathrm{g}$ in each transfection. Transfections were otherwise performed as described (Prendergast et al. 1991) except that the overnight incubation was carried out in $3-4 \% \mathrm{CO}_{2}$ (Chen and Okayama 1987) and plasmid DNAs were purified with a commercial preparatory column (Qiagen, Studio City, $\mathrm{CA}$ ) rather than by $\mathrm{CsCl}$ banding. We observed that these modifications improved the reproducibility of transfection efficiency compared with previous protocols. Foci were scored by methanol fixation and staining with cresyl violet acetate at 1214 days post-transfection. All macroscopically scored foci were examined at low magnification under the microscope to confirm the appearance of transformed cells.

\section{Acknowledgments}

We are grateful to Adrian Ferré-D'Amare and Steven Burley for providing Myn $\Delta \mathrm{C}-$ Sepharose and advice on the experiment in which it was used. We thank Greg Kato, Chi Dang, Steven Berberich, Michael Cole, Elizabeth Blackwood, and Bob Eisenman for communicating results before publication. For critical comments on the manuscript, we are grateful to Kim Boulukos, Karen Buchkovich, and Doug MacGregor. G.C.P. especially thanks Rick Metz and Kris Prendergast for their support and encouragement during the course of the work. VAX computing was supported by National Science Foundation grant DIR-8908095. G.C.P. was supported by a postdoctoral fellowship from the American Cancer Society. This research was supported by grant CA44042 to E.B.Z. from the National Institutes of Health. R.H. is an associate and E.B.Z. is an investigator of the Howard Hughes Medical Institute.

The publication costs of this article were defrayed in part by payment of page charges. This article must therefore be hereby marked "advertisement" in accordance with 18 USC section 1734 solely to indicate this fact. 


\section{References}

Berberich, S.J. and M.D. Cole. 1992. Casein kinase II inhibits the DNA binding activity of Max homodimers but not Myc/ Max heterodimers. Genes \& Dev. 6: 166-176.

Blackwell, T.K., L. Kretzner, E.M. Blackwood, R.N. Eisenman, and H. Weintraub. 1990. Sequence-specific DNA-binding by the c-Myc protein. Science 250: 1149-1152.

Blackwood, E. and R.N. Eisenman. 1991. Max: A helix-loophelix zipper protein that forms a sequence-specific DNAbinding complex with Myc. Science 251: 1211-1217.

Blackwood, E., B. Lüscher and R.N. Eisenman. 1992. Myc and Max associate in vivo. Genes \& Dev. 6: $71-80$.

Boyle, W.J., T. Smeal, L.H.K. Defize, P. Angel, J.R. Woodgett, M. Karin, and T. Hunter. 1991. Activation of protein kinase C decreases phosphorylation of c-Jun at sites that negatively regulate its DNA-binding activity. Cell 64: 573-584.

Cai, M. and R.W. Davis. 1990. Yeast centromere binding protein CBF1, of the helix-loop-helix protein family, is required for chromosome stability and methionine prototrophy. Cell 61: $434-446$

Chen, C. and H. Okayama. 1987. High efficiency transformation of mammalian cells by plasmid DNA. Mol. Cell. Biol. 7: 2745-2752.

Dang, C., M. McGuire, M. Buckmire and W.M.F. Lee. 1989. Involvement of the "leucine zipper" region in the oligomerization and transforming activity of human c-myc protein. Nature 337: 664-666.

Dang, C.V., J. Barrett, M. Villa-Garcia, L.M.S. Resar, G.J. Kato and E.R. Fearon. 1991. Intracellular leucine zipper interactions suggest c-Myc hetero-oligomerization. Mol. Cell. Biol. 11: $954-962$.

Dang, C.V. and W.M.F. Lee. 1988. Identification of the human c-myc protein nuclear translocation signal. Mol. Cell. Biol. 8: 4048-4054.

- 1989. Nuclear and nucleolar targeting sequences of c-erbA, c-myb, N-myc, p53, HSP70, and HIV tat proteins. $J$. Biol. Chem. 264: 18019-18023.

Dingwall, C. and R.A. Laskey. 1986. Protein import into the cell nucleus. Annu. Rev. Cell Biol. 2: 367-390.

Halazonetis, T.D. and A.N. Kandil. 1991. Determination of the c-Myc DNA binding site. Proc. Natl. Acad. Sci. 88: 61626166.

Hunter, T. and B.M. Sefton 1980. Transforming gene product of Rous sarcoma virus phosphorylates tyrosine. Proc. Natl. Acad. Sci. 77: 1311-1315

Jans, D.A., M.J. Ackermann, J.R. Bischoff, D.H. Beach, and R. Peters. 1991. p34 $4^{\text {cdc2 }}$-mediated phosphorylation at $\mathrm{T}^{124}$ inhibits nuclear import of SV-40 T antigen products. $J$. Cell Biol. 115: 1203-1212.

Jones, N. 1990. Transcriptional regulation by dimerization: Two sides to an incestuous relationship. Cell 61: 9-11.

Kato, G., W.M.F. Lee, L. Chen, and C. Dang. 1992. Max: Functional domains and interaction with c-Myc. Genes \& Dev. 6: 81-92.

Kelekar, A. and M.D. Cole. 1987. Immortalization by c-myc, $\mathrm{H}-\mathrm{ras}$, and Ela oncogenes induces differential cellular gene expression and growth factor responses. Mol. Cell. Biol. 7: 3899-3907.

Kerkhoff, E., K. Bister, and K.-H. Klempnauer. 1991. Sequencespecific DNA-binding by Myc proteins. Proc. Natl. Acad. Sci. 88: 4323-4327.

Land, H., L.F. Parada, and R.A. Weinberg. 1983. Tumorigenic conversion of primary embryo fibroblasts requires at least two cooperating oncogenes. Nature 304: 596-602.

Lüscher, B. and R.N. Eisenman. 1990. New light on Myc and
Myb. Part I. Myc. Genes \& Dev. 4: 2025-2035.

Lüscher, B., E.A. Kuenzel, E.G. Krebs, and R.N. Eisenman. 1989. Myc oncoproteins are phosphorylated by casein kinase II. EMBO I. 8: 1111-1119.

Mäkelä, T.P., P.J. Koskinen, I. Västrik, and K. Alitalo. 1992. Alternative forms of Max as enhancers or suppressors of Myc-Ras cotransformation. Science 256: 373-377.

Prendergast, G.C. and E.B. Ziff. 1989. DNA binding motif /scientific correspondence). Nature 341: 392.

. 1991a. Mbh1: A novel gelsolin/severin-related protein which binds actin in vitro and exhibits nuclear localization in vivo. EMBO I. 10: 757-766.

. 1991b. Methylation-sensitive sequence-specific DNA binding by the c-Myc basic region. Science 251: 186-189.

. 1992. A new bind for Myc. Trends Genet. 8: 91-96.

Prendergast, G.C., D. Lawe and E.B. Ziff. 1991. Association of Myn, the murine homolog of Max, with c-Myc stimulates methylation-sensitive DNA binding and Ras cotransformation. Cell 65: 395-407.

Rihs, H.-P., D.A. Jans, H. Fan, and R. Peters. 1991. The rate of nuclear cytoplasmic protein transport is determined by the casein kinase II site flanking the nuclear localization sequence of the SV40 T-antigen. EMBO J. 10: 633-639.

Smith, M.J., D.C. Charron-Prochownik, and E.V. Prochownik. 1990. The leucine zipper of c-Myc is required for full inhibition of erythroleukemia differentiation. Mol. Cell. Biol. 10: $5333-5339$.

Stone, J., T. de Lange, G. Ramsay, E. Jakobovits, J.M. Bishop, H. Varmus, and W. Lee. 1987. Definition of regions in human $\mathrm{c}-\mathrm{myc}$ that are involved in transformation and nuclear localization. Mol. Cell. Biol. 7: 1697-1709.

Wenzel, A., C. Cziepluch, U. Hamann, J. Schürmann and M. Schwab. 1991. The amino-Myc oncoprotein is associated in vivo with the phosphoprotein $\operatorname{Max}(\mathrm{p} 20 / 22)$ in human neuroblastoma cells. EMBO I. 10: 3703-3712. 


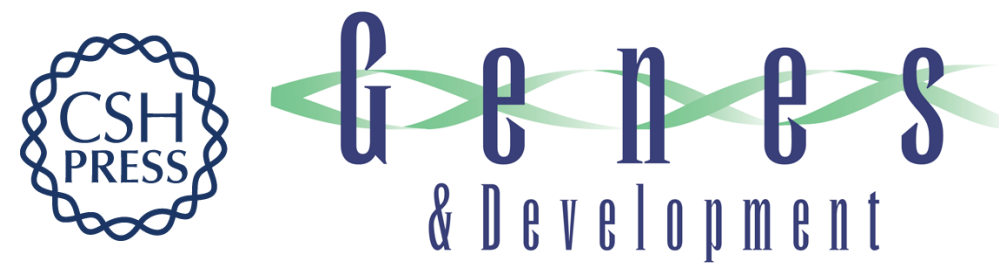

\section{Biphasic effect of Max on Myc cotransformation activity and dependence on amino- and carboxy-terminal Max functions.}

G C Prendergast, R Hopewell, B J Gorham, et al.

Genes Dev. 1992, 6:

Access the most recent version at doi:10.1101/gad.6.12a.2429

References This article cites 32 articles, 19 of which can be accessed free at:

http://genesdev.cshlp.org/content/6/12a/2429.full.html\#ref-list-1

License

Email Alerting

Service

Receive free email alerts when new articles cite this article - sign up in the box at the top right corner of the article or click here.

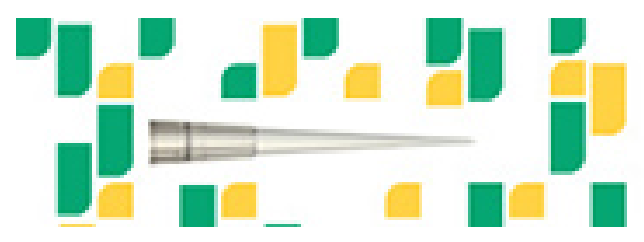

Focused on your science. 